\title{
Epithelia-derived wingless regulates dendrite directional growth of drosophila ddaE neuron through the Fz-Fmi-Dsh-Rac1 pathway
}

Xiaoting $\mathrm{Li}^{1,2+}$, Yan Wang ${ }^{3+}$, Huan Wang ${ }^{1,2}$, Tongtong Liu ${ }^{1,2}$, Jing Guo ${ }^{1}$, Wei Yi ${ }^{1}$ and Yan $\mathrm{Li}^{1 *}$

\begin{abstract}
Background: Proper dendrite patterning is critical for the receiving and processing of information in the nervous system. Cell-autonomous molecules have been extensively studied in dendrite morphogenesis; however, the regulatory mechanisms of environmental factors in dendrite growth remain to be elucidated.

Results: By evaluating the angle between two primary dendrites (PD-Angle), we found that the directional growth of the primary dendrites of a Drosophila periphery sensory neuron ddaE is regulated by the morphogen molecule Wingless (Wg). During the early stage of dendrite growth, Wg is expressed in a group of epithelial cells posteriorly adjacent to ddaE. When Wg expression is reduced or shifted anteriorly, the PD-Angle is markedly decreased. Furthermore, Wg receptor Frizzled functions together with Flamingo and Dishevelled in transducing the Wg signal into ddaE neuron, and the downstream signal is mediated by non-canonical Wnt pathway through Rac1.

Conclusions: In conclusion, we reveal that epithelia-derived Wg plays a repulsive role in regulating the directional growth of dendrites through the non-canonical Wht pathway. Thus, our findings provide strong in vivo evidence on how environmental signals serve as spatial cues for dendrite patterning.
\end{abstract}

Keywords: Epithelia-derived Wingless, Dendrite directional growth, Frizzled, Flamingo, non-canonical Wnt pathway

\section{Background}

Dendrites receive and process most of the information from external stimuli and input neurons; therefore, it is essential for dendrites to develop an elaborate arborization pattern. Both cell-intrinsic and extrinsic factors play instructive roles in regulating dendrite development [1]. A number of intrinsic factors, such as transcriptional factors, organelles, and regulators of cytoskeleton, provide internal forces for dendrite growth and branching [2, 3]. On the other hand, extrinsic factors from the environment serve as spatial cues to guide dendrites to their targeting areas and ensure the extension direction and branching pattern. In mammalian cortical plate, several extrinsic factors, including Semaphorin3A (Sema3A), Slit, Brain-derived

\footnotetext{
* Correspondence: liyan@ibp.ac.cn

${ }^{\dagger}$ Equal contributors

'State Key Laboratory of Brain and Cognitive Science, Institute of Biophysics,

Chinese Academy of Sciences, Beijing 100101, China

Full list of author information is available at the end of the article
}

neurotrophic factor (BDNF), and Notch, are coordinated to direct the growth of apical dendrites towards the pial surface, and thus control the dendrite patterning in pyramidal cells [4-7]. In C. elegans, two epithelial adhesion molecules, SAX-7 and MNR, and a neuronal receptor, DMA-1, form a tripartite ligandreceptor complex to provide spatial information for dendrite branching in the PVD neurons $[8,9]$. Similarly, in Drosophila, several extrinsic factors have been reported to guide the dendrites to their targeting sites, including Sema1A, Netrin and Slit $[10,11]$. These earlier studies indicated that environmental cues play essential roles in regulating the directional growth of dendrites in Drosophila.

Drosophila dendritic arborization (da) neurons extend their dendrites in a 2D plane at the basal surface of the epidermis in contact with extracellular matrix (ECM) 
$[12,13]$. These da neurons are classified into four classes, according to their stereotyped dendritic fields and branching complexities, and have been used as an ideal model system for studying dendrite morphogenesis [3]. It has been reported that extrinsic factors originating from the ECM and epithelium adjacent to da neurons regulate the tiling, scaling and self-avoidance processes of class IV da neurons [12-16]. These factors play essential roles in establishing and maintaining the radial dendrite pattern of class IV neurons, therefore ensuring that the neurons cover the whole body wall of larvae. Different from class IV neurons, class I da neurons possess comb-like dendritic arborizations, with their primary and secondary dendrites extending along the dorsalventral (DV) and the anterior-posterior (AP) directions, respectively. A study from $T$. Uemura's lab indicated that Ten-m, a homophilic cell adhesion molecule of the Teneurin family, is highly expressed in the class I neuron ddaE, where it was found to regulate directional control of dendritic sprouting and extension through homodimer interactions with epidermal Ten-m molecules. The secondary dendrites of the ddaE neuron respond to the Ten-m gradient along the AP direction in epidermis, with the consequence of realizing posterior-oriented comb-like pattern [17]. Notably, primary dendrites of the ddaE neuron extend along the DV direction, which is unlikely affected by the Ten-m signal. Whether its directional growth is also regulated by environmental cues remains uninvestigated.

During embryonic development of Drosophila, several morphogens are secreted from the epithelium, providing critical spatial information to govern the morphogenesis of tissues. One morphogen, Wingless (Wg), also known as Drosophila Wnt-1, belongs to the Wnt protein family. Wnt proteins have been studied in great detail for their evolutionarily conserved roles in cell fate specification, axon guidance, and synapse formation during the development process of the nervous system [18-20]. Particularly, the Wnt signal has been found to function as an environmental cue in regulating the directional growth of axons, and Frizzled (Fz) and Derailed (Drl)/Ryk receptors have been suggested to mediate attractive and repulsive roles, respectively, in Wnt signaling [21-23]. It has been reported that during dendrite development, Wnt proteins promote dendritic branching and outgrowth in cultured hippocampal neurons of mice as well as in sensory neurons of C. elegans [24, 25]. However, whether Wnt signals also function as spatial cues to regulate directional growth of dendrites remains unclear.

In this study, we used the Drosophila ddaE neuron as an in vivo model to study the directional growth of dendrites. We found that epithelia-derived morphogen Wg functions as a repulsive cue to regulate the growth direction of primary dendrites in ddaE neuron.

\section{Results}

The PD-Angle of the ddaE neuron is decreased when $\mathrm{Wg}$ expression is reduced in the adjacent epithelial cells To study whether the primary dendrites of the ddaE neuron also develop according to the environmental signals, we examined the localization of epithelia-derived signals relative to the ddaE neuron at the early dendritogenesis stage, i.e. embryonic stage 13-14. Using $w g$-Gal4; UASmGFP embryos, we found that in the dorsal region of the abdominal segments 4-6 (AS4-6), Wg was expressed in a small group of epithelial cells adjacent to the dorsal cluster of da neurons (Fig. 1a); however, as shown in the X-Z or Y$\mathrm{Z}$ section images, $\mathrm{Wg}$ expression was not detected in those neurons (Fig. 1a'). We set the coordinate system with the position of the ddaE soma as the origin, the AP direction as the $\mathrm{X}$ axis, and the DV direction as the $\mathrm{Y}$ axis. In this coordinate system, the Wg-expressing cells were mainly localized in the first, i.e. dorsal-posterior quadrant (Fig. 1b). The adjacent localization of Wg expressing cells and the ddaE neuron raises the possibility that Wg functions as a spatial cue to regulate the dendrite patterning of the ddaE neuron.

To determine whether epithelial Wg has an effect on the dendrite growth of ddaE neuron, we used an amorphic mutant $w g^{l-12}$, the $\mathrm{Wg}$ protein of which has a single amino acid change (C104S), resulting in temperature-sensitive deficiency of Wg secretion [26, 27]. To visually display the direction of dendrite extension, we traced the primary dendrites of ddaE neurons and drew a circle around the soma with a radius of $20 \mu \mathrm{m}$, and the intersections of primary dendrites and the circle were marked in the AP-DV coordinate system (Fig. 1c). In wild type flies, the intersections of all dorsal primary dendrites were located in the two dorsal quadrants, and those of ventral primary dendrites were mainly located in the ventral-posterior quadrant. In $w g^{l-12}$ mutant flies, the distribution of dorsal intersections showed little change when compared to wild type flies; however, the ventral intersections greatly shifted towards the AP axis, with a considerable number of those being located in the dorsal-posterior quadrant. To quantify this change in growth direction, we defined the angle between the initial $20 \mu \mathrm{m}$ of two primary dendrites as the PD-Angle. As expected, the PD-Angle was markedly decreased in the $w g^{l-12}$ mutant relative to that found in control larvae (Fig. 1d-e). Therefore, these results suggested that epithelial Wg provided a repulsive signal for the directional growth of dendrites.

Dendritogenesis of class I da neurons is initiated during embryonic stage, and the dendrite pattern is stabilized at early larval stages, followed by scaling up during larval development $[15,28]$. We thus wondered whether Wg signal affected the directional growth of ddaE neuron from early stages of dendrite development. The main structure of primary dendrites of ddaE neuron is formed by embryonic 


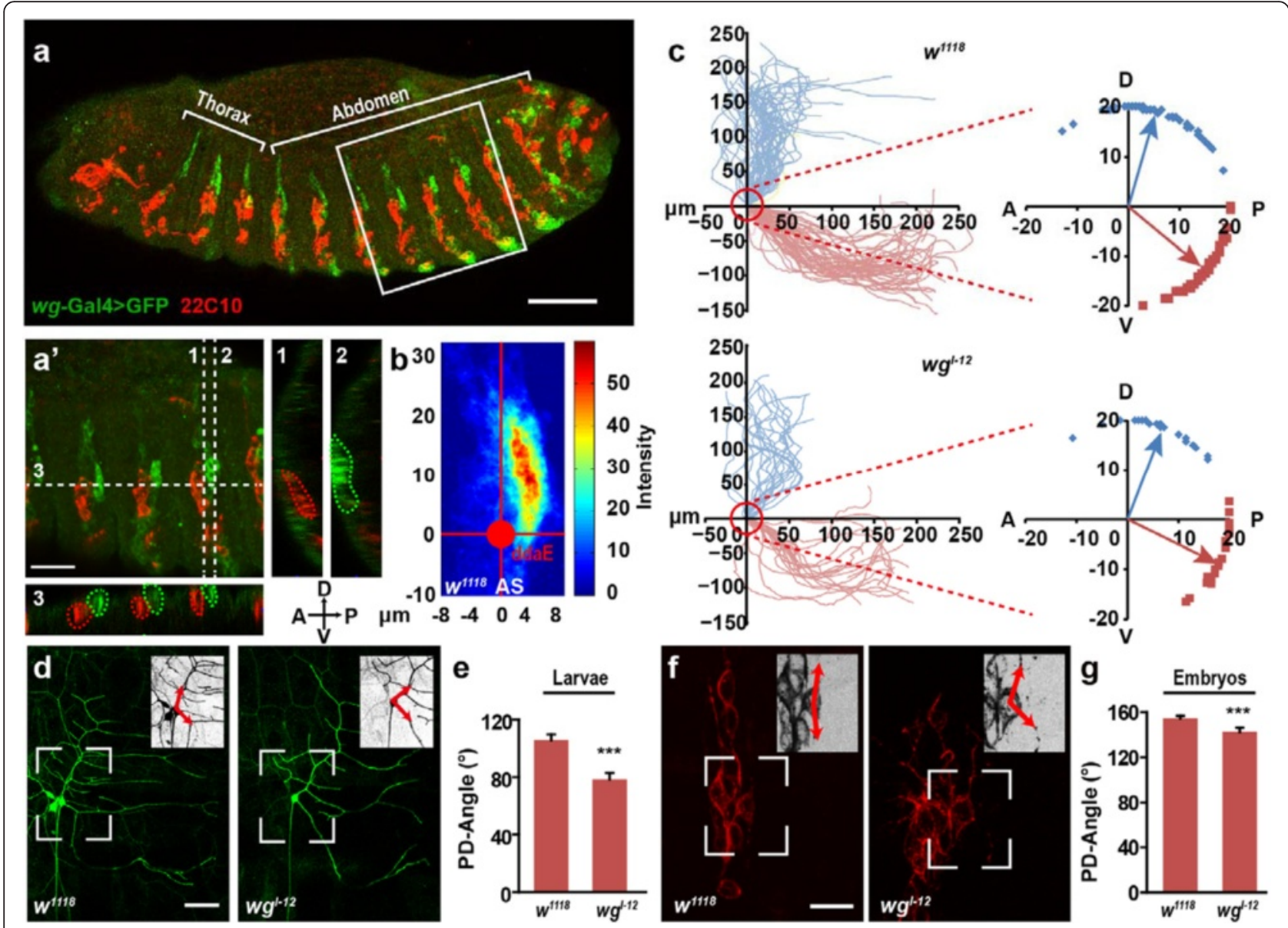

Fig. 1 Directional growth of primary dendrites in ddaE neuron is deficient in $\mathrm{wg}^{1-12}$ mutant. a Wg is expressed at the posterior side of the dorsal cluster of da neurons in whole mount fly embryos. Wg expression pattern (green) is monitored by UAS- mCD8-eGFP under the control of wg-Gal4. The da neurons (red) are stained by antibody 22C10. White brackets indicate the regions of thorax and abdomen. $\mathbf{a}^{\prime}$, Enlarged view of AS4-6 (white square in a). White broken lines 1-3 indicate the positions of X-Z or Y-Z section images 1-3, respectively. Red broken lines indicate the ddaE neurons and green broken lines indicate the Wg-expression cells. b Pseudo-color image shows that Wg-expressing cells are localized mainly in the dorsal-posterior region relative to ddaE neuron in $w^{1718}$ AS. c The initial $20 \mu \mathrm{m}$ parts of primary dendrites shift towards AP axis in $\mathrm{wg}^{l-12}$ mutants. Left panels show the trajectory of primary dendrites of ddaE in the $3^{\text {rd }}$ instar larvae. Red circles indicate the circle around the soma with a radius of $20 \mu \mathrm{m}$. The enlarged view of the intersections of the circle and the trajectory of primary dendrites are shown in right panels. Blue dots and red dots indicate the dorsal intersections and ventral ones, respectively. The Blue arrows and red arrows indicate the average direction of dorsal and ventral primary dendrites, respectively. A, P, D and V indicates the direction of anterior, posterior, dorsal and ventral, respectively. $(n=24-50)$. d-e PD-Angle significantly decreased in $w^{l-12}$ mutants. $\mathbf{d}$ Representative images of ddaE neurons in wild type $w^{1118}$ and $w g^{1-12}$ mutant at third instar larvae stage. Gal4 ${ }^{2-21}$, UAS-mCD8-eGFP was used to visualize the entire neuron. e Quantification statistic of PD-Angle in wild type $w^{1718}$ and wg ${ }^{1-12}$ mutant. $(\mathrm{n}=24-50) . \mathbf{f}-\mathbf{g}$ The PD-Angle is decreased in wg $^{l-12}$ mutants at embryonic stage 17. $(\mathrm{n}=35-57)$. Scale bars, $50 \mu \mathrm{m}$ in $(\mathbf{a}$ and $\mathbf{d})$ and 20 $\mu \mathrm{m}$ in ( $\mathbf{a}^{\prime}$ and $\left.\mathbf{f}\right)$. In all figures, representative images are shown in anterior left and dorsal up; white broken rectangles indicate the position of the inset in the upper-right corner, and red arrows indicate the initial growth direction of the two primary dendrites. ${ }^{*} p<0.05,{ }^{* *} p<0.01,{ }^{* * *} p<0.001$, and N.S. indicates no significant changes

stage 17. Thus, we examined the dendrite morphology of $\mathrm{ddaE}$ at this stage and observed a decrease in the PDAngle in $w g^{l-12}$ mutant flies (Fig. 1f-g). These results suggested that the change of PD-Angle in the third instar larval stage was formed at the embryonic stage, during which the Wg signal regulates the directional growth of the primary dendrites, especially the initial parts.

We then examined the expression pattern of $\mathrm{Wg}$ in $w g^{l-12}$ mutant at both mRNA and protein levels by fluorescent in situ hybridization (FISH) and antibody immunostaining, respectively. The $w g$ mRNA expression was characterized using two parameters, the width of $w g$ distribution region ( $w g$ distribution) and the distance between the center of $w g$ distribution and the posterior segmental boundary (Center-to-boundary Distance). To exclude the individual difference in segmental width, we normalized the two parameters to the segmental width in each embryo. Compared to wild type embryos, neither the distribution nor the Center-to-boundary Distance of $w g$ mRNA differed in $w g^{l-12}$ mutant embryos, 
suggesting that in this mutant, $w g$ is expressed in the same group of epithelial cells as those in wild type flies (Fig. 2a-b). To check whether same results apply to Wg protein, we performed Wg antibody immunostaining experiment. As shown in Fig $2 \mathrm{c}-\mathrm{d}$, in $w g^{l-12}$ mutants, however, Wg expression levels were significantly lower, while the Center-to-boundary Distance remained unchanged.
This result is consistent with previous reports indicating that the mutation of a signal amino acid in $w g^{l-12}$ mutant affects the protein stability of Wg but not $w g$ mRNA transcription.

To examine whether the dendrite phenotype of ddaE neuron in $w g^{l-12}$ mutant was affected by reduced Wg expression, we used RNA interference to interrupt $\mathrm{Wg}$
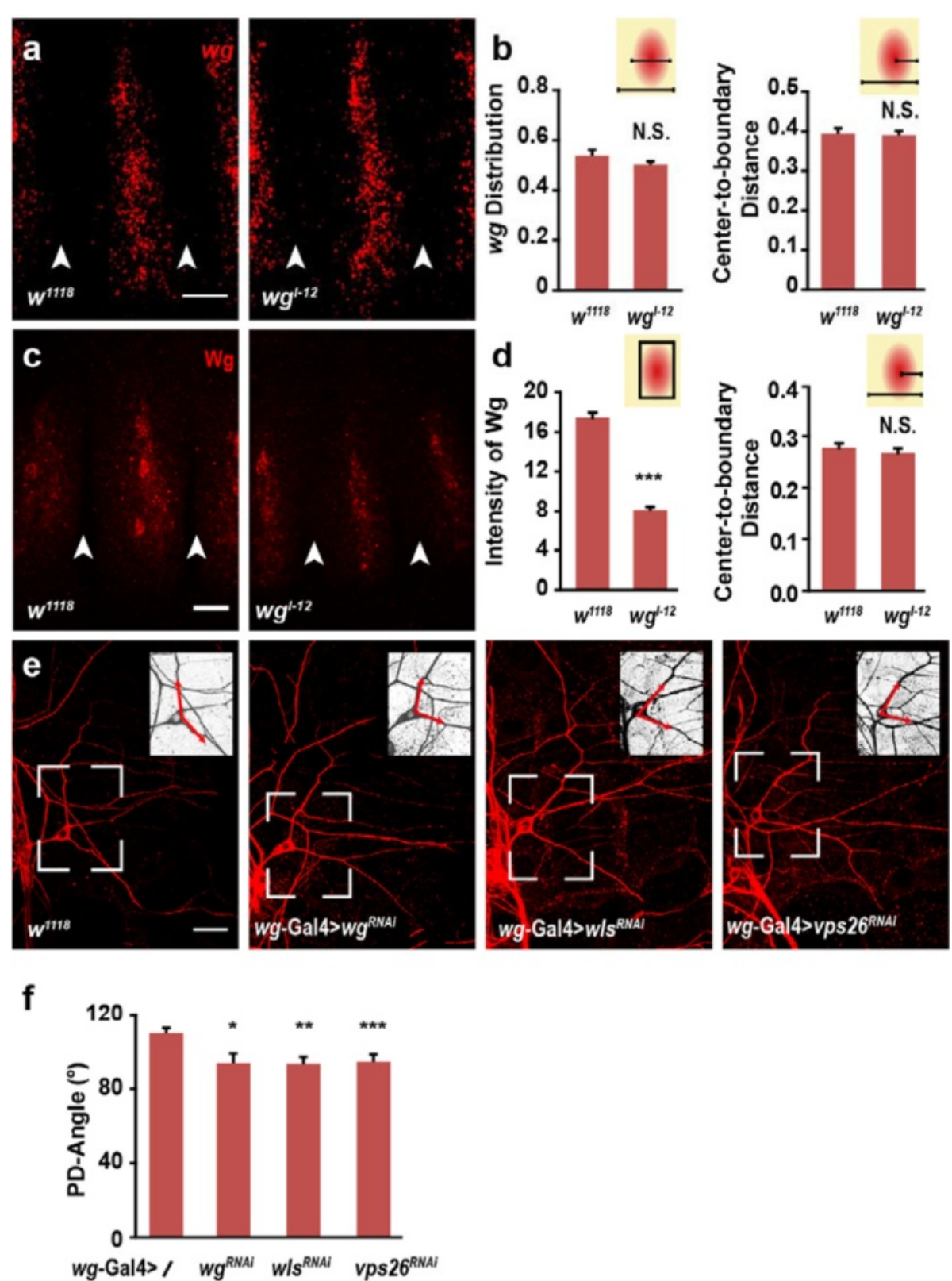

Fig. 2 Down regulation of environmental Wg induces the decrease of PD-Angle. a-b At embryonic stage 14, wg RNA is detected by FISH. Normalized to the segment width, both wg RNA distribution and the distance between the center of wg signal and posterior segmental boundary are comparable to wild type control. White arrow heads in (a) indicate the segment boundaries. The insert cartoons in (b) represent the expressing region of $\mathrm{Wg}$ with red oval, the segment region with yellow rectangle, and the parameters quantified in corresponding panels. $(n=15-30)$. c-d Wg expression level is significantly decreased in wg $^{1-12}$ embryos, while its center-to-boundary distance is not changed after normalized to the segment boundary. $(n=28-32)$. e-f Knockdown of $w g$, wntless or vps26 in epithelial cells by wg-Gal4 results in the decreased PD-Angle. ( $n=22-49)$. Scale bars, $10 \mu \mathrm{m}$ in (a) and (c) and $25 \mu \mathrm{m}$ in (c) 
signal in wild type flies. Knockdown of $w g$ by expressing $w g^{R N A i}$ in epithelial cells (with $w g$-Gal4) resulted in a decrease in PD-Angle. In contrast, it remained unaffected when $w g^{R N A i}$ was expressed in $\mathrm{ddaE}$ neurons with Gal4 ${ }^{2-21}$ (Fig. 2e-f and Additional file 1: Fig. S1). Previous studies indicated that Wntless (Wls) and Vps26 play important roles in Wg secretion process. Downregulation of Wls and Vps26 results in the accumulation of Wg in the cytoplasm [29, 30]. We found that knockdown of Wls and Vps26 using wg-Gal4 resulted in the decreased PD-Angle of ddaE neuron (Fig. 2e-f). These results indicated that down regulating the expression level of Wg or inhibiting the secretion of Wg leads to a more posterior extension of the initial parts of primary dendrites. Taken together, epithelia-derived Wg is posteriorly adjacent to the primary dendrites of ddaE neuron, and reduced Wg signal leads to a significant reduction in the PD-Angle.

\section{Anterior-shifted $\mathbf{W g}$ also results in dendrite directional growth defect in ddaE neuron}

When we examined the PD-Angle of ddaE neurons in late embryos or $3^{\text {rd }}$ instar larvae of wild type flies, we noted that in the thoracic segments (TS) of wild type flies, the PD-Angle of ddaE neuron was significantly smaller than that in AS (Fig. 3a-b). To investigate whether this difference is also due to decreased Wg signals as that in the $w g^{l-12}$ mutant, we next examined the $\mathrm{Wg}$ expression patterns in the TS using $w g$-Gal4;UAS-mGFP embryos. We noticed that relative to the Wg-expression pattern in
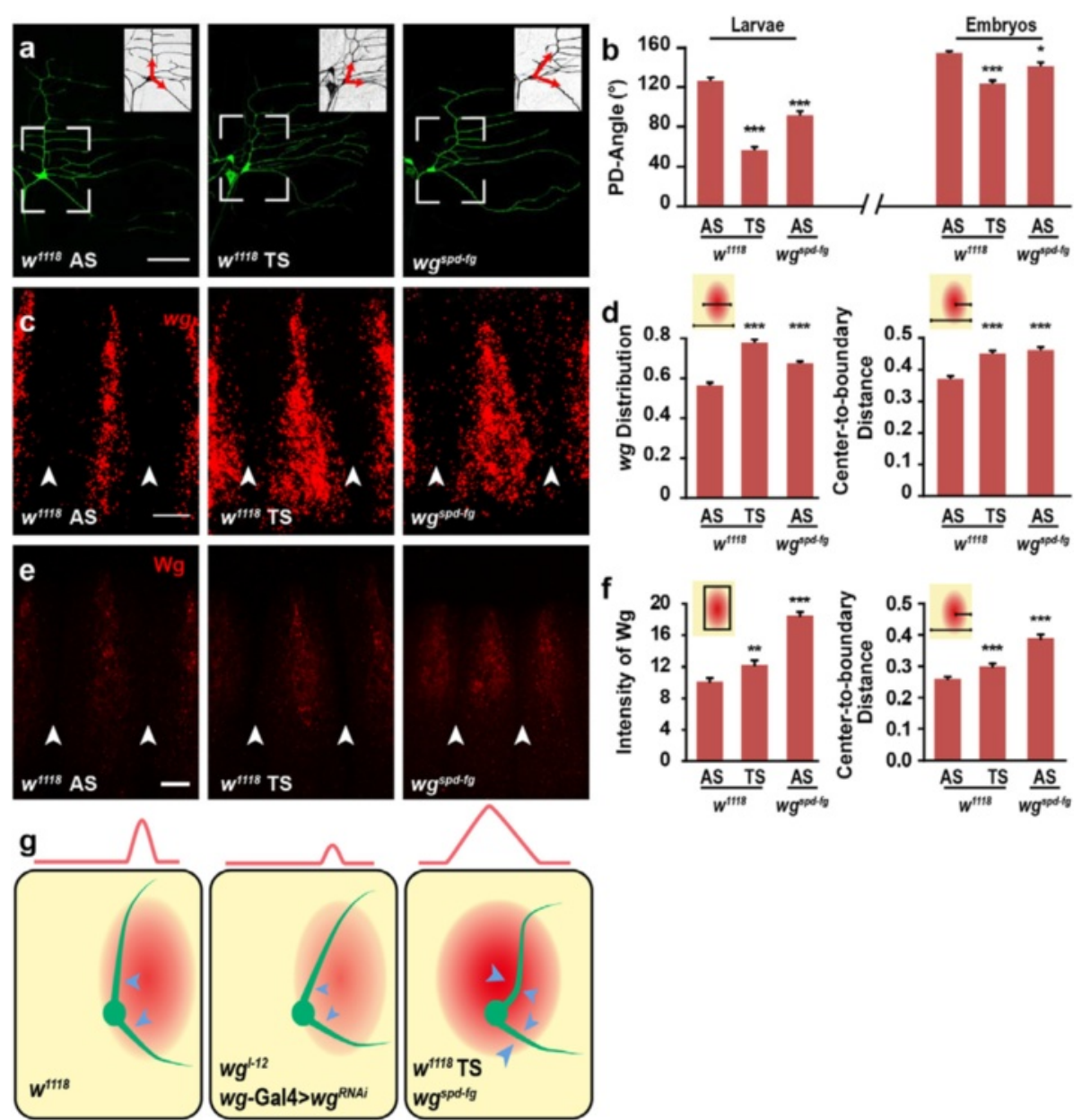

Fig. 3 Anterior-shifted Wg expression results in a reduced PD-Angle. a-b The PD-Angle is significantly decreased in TS and wg $g^{\text {spd-fg }}$ in both late embryos and third instar larvae. ( $n=26-57)$. $\mathbf{c}-\mathbf{d}$ wg expression region is significantly wider in both wild type TS2-3 and $w g^{\text {spd-fg }}$ AS4-6, when compared to wild type AS4-6. The distance between the center of wg signal and posterior segmental boundary is also significantly increased when normalized to segment width. $(n=20-29)$. e-f Wg expression is markedly increased in TS2-3 of wild-type $\left(w^{1118}\right)$ and in AS4-6 of $w g^{\text {spd-fg }}$ mutants, and the center of Wg signal is also significantly anteriorly shifted. $(n=23-36)$. $\mathbf{g}$ Schematic diagram of Wg expression pattern and dendrite orientation. The ddaE neuron is located at the anterior side and adjacent to Wg-expressing cells, and the two primary dendrites are orientated towards the posterior when Wg expression is reduced (as in $w g^{l-12}$ and $w g$-Gal $4>w g^{R N A)}$ ), or when Wg is ectopically expressed in the anterior region (as in $w g^{\text {spd-fg }}$ and wild-type TS). Scale bar: $50 \mu \mathrm{m}$ in (a) and $10 \mu \mathrm{m}$ in (c and e) 
AS, Wg-expressing cells in TS displayed a considerable towards the anterior direction (Additional file 2: Fig. S2a). In agreement with this observation, FISH results indicated that $w g$ mRNA in TS was detected in a wider region, and the center of $w g$ mRNA signal was shifted towards the anterior direction in stage 13-14 embryos, as indicated by the increased Center-to-boundary Distance when normalized to the segmental width (Fig. 3c-d). Using immunostaining, we found that Wg expression levels were considerably high in TS (Fig. 3e-f). In addition, normalized to segment width, the Center-to-boundary Distance was significantly higher in TS compared to that in AS (Fig. 3f), suggesting that Wg protein was located further towards the anterior end of the TS segment. Together, these results indicated that the PD-Angle is smaller in TS, which might due to the anterior-shifted Wg expression.

To test this possibility, we searched for $w g$ mutants with anterior-shifted Wg expression and found a $w g$ mutant, namely $w g^{s p d-f g}$. Using immunostaining and FISH, we found that in stage 13-14 embryos, Wg was expressed at a higher level and in a more anterior region in AS of $w g^{\text {spd-fg }}$ mutant compared to wild type embryos. The Wg expression pattern in $w g^{s p d-f g}$ mutant is similar to that in TS of wild type flies (Fig. 3c-f). The $w g^{s p d-f g}$ mutant contains a $1.2 \mathrm{~kb}$ deletion in the enhancer region of the $w g$ gene [31]. Thus, we generate the $w g^{s p d-f g}$-Gal4 transgenic flies to mimic the Wg expression pattern of $w g^{\text {spd-fg }}$ mutant flies. Using GFP as a reporter, we found that in AS4-6 segments of stage 14 embryos, the expression patterns of $w g^{s p d-f g}$-Gal4 shifted anteriorly relative to the soma of the ddaE neuron, when compared with $w g^{w t}$-Gal4 (Additional file 2: Fig. S2b-c). Together with the results obtained from antibody staining and FISH, we demonstrated that the $w g^{\text {spd-fg }}$ mutant is a $w g$ mutant exhibiting anterior-shifted Wg expression, similar to that in TS of wild type flies. In agreement with the result that the PD-Angle of the ddaE neuron is decreased in TS of wild type flies, the PD-Angle of ddaE neuron in $w g^{s p d-f g}$ mutant was also significantly decreased at both embryonic and the $3^{\text {rd }}$ instar larva stage (Fig. 3a-b and Additional file 3: Fig. S3a). Together, these results indicated that the anterior-shifted $\mathrm{Wg}$ expression also leads to a decrease in the PD-Angle in both the TS of wild type and the $w g^{s p d-f g}$ mutant.

To further examine whether the abnormal directional growth affects the coverage field of ddaE neuron, we investigated the dendrite coverage area outlined either by the two primary dendrites (PD-Area) or by all branch tips (Total area) in wild type and $w g^{s p d-f g}$ mutant. Both PD-Area and Total area of ddaE neuron were significantly reduced in $w g^{s p d-f g}$ mutant (Additional file 3: Fig. S3b). The coverage range in the DV direction was significantly shorter in $w g^{s p d-f g}$ mutant relative to wild type, but was the same in the AP direction (Additional file 3: Fig. S3c). This suggests that the observed reduction in coverage area was a consequence of the reduction in DV range. Furthermore, the total length of two primary dendrites (PD-total length) was comparable between $w g^{\text {spd-fg }}$ mutant and wild type (Additional file 3: Fig. S3d), suggesting that the decreased coverage area was not caused by general growth defect, but instead by directional growth defect. Together, these findings indicate that the primary dendrites provide the frame of dendritic patterning in ddaE neuron, thus directional growth of primary dendrites significantly contributes to the control of dendrite occupation.

Taken together, our results provide an in vivo evidence that Wg signal plays an essential role in regulating the directional growth of primary dendrites in ddaE neuron. $\mathrm{Wg}$ is secreted from a small group of epithelial cells posteriorly adjacent to the ddaE neuron. When Wg expression is reduced (as in $w g^{l-12}$ and $w g-G a l 4>w g^{R N A i}$ ) or Wg secretion is deficient ( $w g-G a l 4>w l s^{R N A i}$ and $w g$ Gal4 > vps $26^{R N A i}$ ), the extracellular levels of Wg are decreased in the posterior region of the ddaE neuron, resulting in an extension of the two primary dendrites of ddaE neuron to a more posterior position. When the Wg-expressing region is anteriorly shifted (as in $w g^{s p d-f g}$ and TS of wild type), the extracellular Wg levels are increased, especially in the anterior side of the ddaE neuron, and this change also results in the posteriorlyoriented primary dendrites of the ddaE neuron (Fig. 3g). Thus, we hypothesized that the epithelia-derived $\mathrm{Wg}$ signal plays a repulsive role in controlling primary dendrite routing of the ddaE neuron. Down-regulation of Wg expression reduces the repulsive power from the posterior side of the ddaE neuron, whereas anteriorlyshifted Wg expression gives the dendrites an anterior to posterior repulsion. Both situations lead to a more posterior directing of the primary dendrites. Furthermore, our results suggest that in neurons with asymmetric dendrite tree like ddaE, the directional growth of the primary dendrites is critical for establishing proper receptive field and thus needs to be precisely regulated.

\section{Frizzled and Flamingo are required for $\mathrm{Wg}$ signal-mediated dendrite routing}

To further investigate how the Wg signal is conveyed into the ddaE neuron, we tested whether the downstream molecules of $\mathrm{Wg}$ signal pathway participate in the regulation of the dendrite development. Fz is a classic Wg receptor that plays an essential role in cell polarity and embryonic development [32]. Using immunostaining, we detected the expression of $\mathrm{Fz}$ in ddaE neuron in both embryos and the $3^{\text {rd }}$ instar larvae (Fig. 4a and Additional file 4: Fig. S4a). We then employed a $f z$ mutant, $f z^{E Y 03114}$, to 

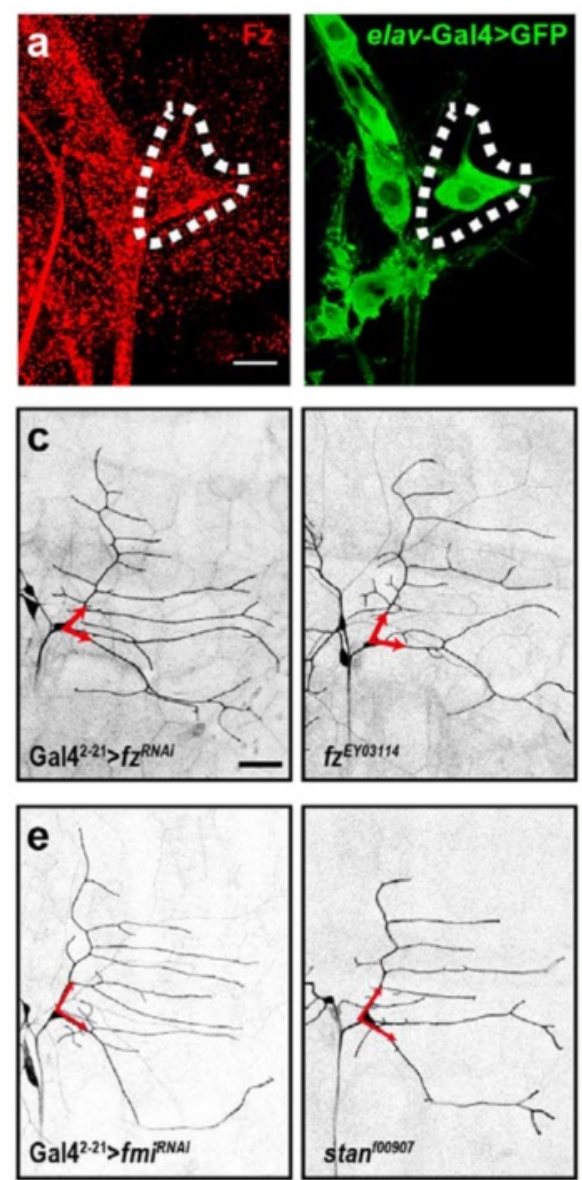

g

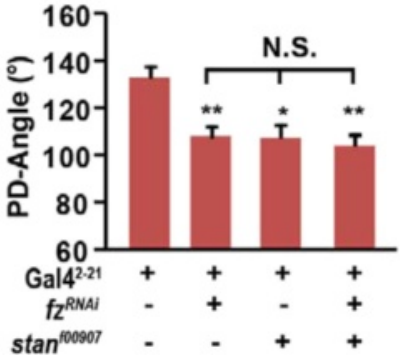

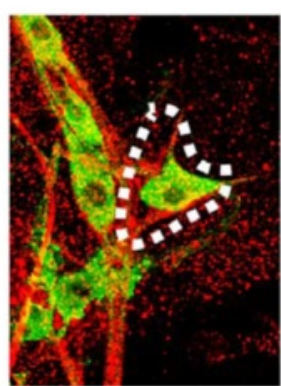
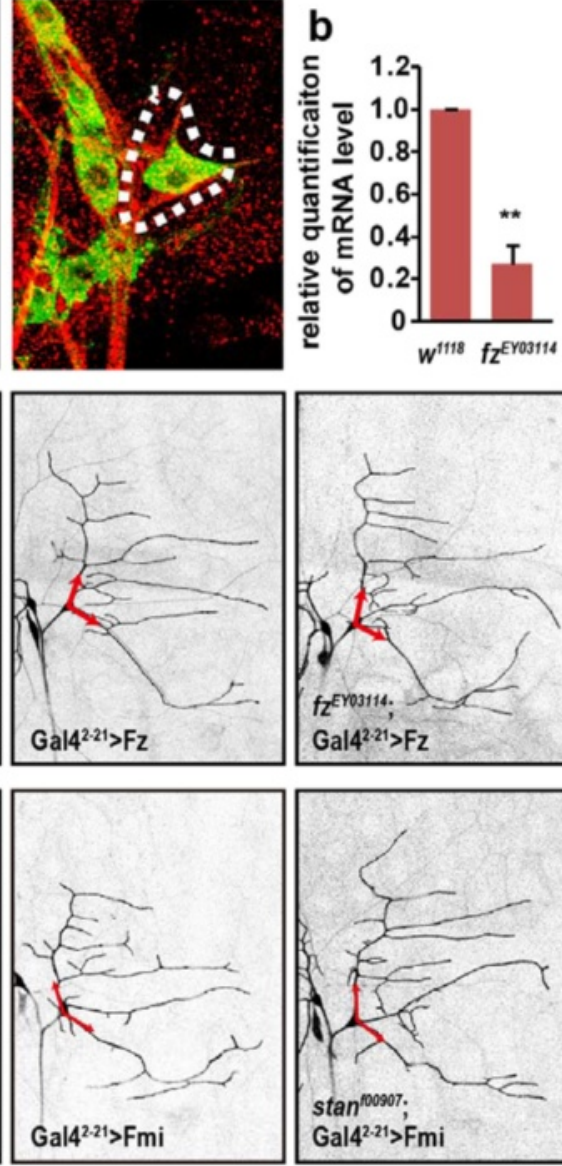

h
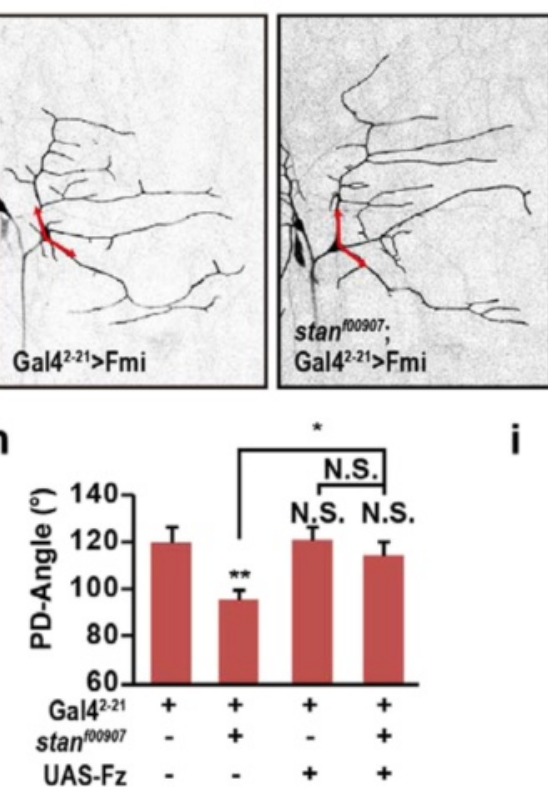

i
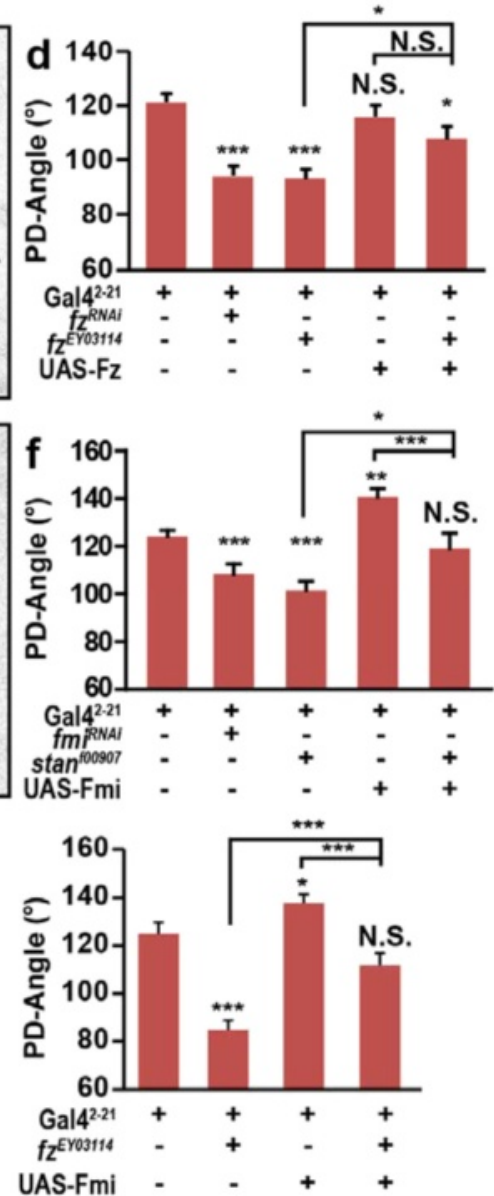

Fig. 4 Frizzled and flamingo are required by the ddaE neuron for dendrite directional growth. a Fz is expressed in ddaE neuron in the $3^{\text {rd }}$ instar larva. Fz is labeled by Fz antibody (red) and neurons are labeled by elav-Gal4 (green). White dot lines show the soma of ddaE neuron. $\mathbf{b} f z$ expression

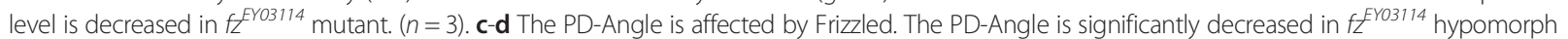
mutant and neural $f^{R N A i}$ expression larvae. Overexpression of $\mathrm{Fz}$ in ddaE neuron does not result in an increase of the PD-Angle. Overexpression of Fz in ddaE neuron in $f^{\text {FOO3114 }}$ mutant background slightly increased the PD-Angle. $(n=50-79)$. e-f The PD-Angle is affected by Fmi. Knockdown of fmi in

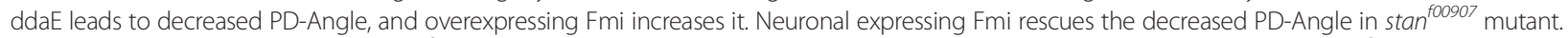
$(n=26-50)$. g Knockdown of $f z$ in a stan ${ }^{\text {f00907 }}$ mutant background does not further decrease the PD-Angle when compared to stan $^{\text {f00907 }}$ mutant or neural knocking down of $f z$. $(n=39-42)$. h Overexpression of Fz in a staf ${ }^{f 00907}$ mutant background rescues the decreased PD-Angle in sta ${ }^{f 00907}$ mutant to the wild type degree. $(n=32-49)$. i PD-Angle of ddaE neuron with neural overexpression of Fmi in $f^{E Y 03114}$ mutant background is comparable to the wild type control. $(n=26-32)$. Red arrows indicate the initial parts of primary dendrites. Scale bar: $10 \mu \mathrm{m}$ in (a) and $50 \mu \mathrm{m}$ in (c)

study the function of $\mathrm{Fz}$ in regulating dendrite directional growth. In $f z^{E Y O 3114}$ mutant, a p-element was inserted into the intron of $f z$ gene region and the mRNA level of $f z$ was significantly decreased as measured by qRT-PCR (Fig. 4b) [33]. Similar to observations in $w g$ mutants, a significant decrease of the PD-Angle was found in the $f z^{E Y 03114} \mathrm{mu}-$ tant. In addition, when $f z$ was knocked down in the neurons by $\mathrm{Gal}_{4}^{2-21}$, the PD-Angle was also significantly reduced. Although overexpression of $\mathrm{Fz}$ by $\mathrm{Gal}_{4}^{2-21}$ showed no increase in PD-Angle in the ddaE neuron, 
overexpression of $\mathrm{Fz}$ in $f z^{E Y 03114}$ mutant partially rescued the mutant phenotype (Fig. $4 \mathrm{c}-\mathrm{d}$ ). These results indicated that $\mathrm{Fz}$ is required in the ddaE neuron for directional growth during dendrite elaboration.

The Ryk receptor family has emerged as a partner of Wnt in regulating developmental events, like axon guidance [34-36]. We thus examined three members in Drosophila Ryk family, Drl, Derailed 2 (Drl-2), and Doughnut (Dnt). The PD-Angle of ddaE neuron was unchanged in any of these mutants or neuronal knockdown larvae (Additional file 5: Fig. S5a-d), indicating that these Ryk receptors are not required for transducing Wg signal in terms of regulating the directional growth of primary dendrites in ddaE neuron.

As an atypical cadherin possessing seven-pass transmembrane receptor features, Fmi is co-localized with $\mathrm{Fz}$ and plays an essential role in the PCP pathway $[37,38]$. It has been found that Fmi is expressed in the epithelial cells, as well as the da neurons, including their dendrites, to regulate dendrite extension $[39,40]$. We adopted two homozygous $f m i$ viable mutants, $\operatorname{stan}^{f 00907}$ and $\operatorname{stan}^{f r z 3}$, which exhibited reduced Fmi expression in both neurons and epithelial cells (Additional file 6: Fig. S6a) [40, 41]. In these fmi mutants, the PD-Angle were significantly decreased, suggesting that similar to $\mathrm{Wg}$ and $\mathrm{Fz}, \mathrm{Fmi}$ is also required for dendrite directional growth (Fig. $4 \mathrm{e}-\mathrm{f}$ and Additional file 6: Fig. S6b-c). In addition, neuronal knockdown of $f m i$ with $\mathrm{Gal} 4^{2-21}$ also induced a reduction of PD-Angle, while overexpressing Fmi in the neuron significantly increased the PD-Angle. Furthermore, neuronal expression of Fmi in a $\operatorname{stan}^{f 00907}$ mutant background fully rescued the decreased PD-Angle in the mutant (Fig. 4e-f), indicating that Fmi plays an essential role in ddaE neuron for regulating dendrite routing.

As both $\mathrm{Fz}$ and $\mathrm{Fmi}$ were expressed in da neurons at the embryonic stage 14 (Additional file 4: Fig. S4b), we asked whether $\mathrm{Fz}$ and Fmi cooperate in dendrite directional growth. Thus we performed genetic interaction experiments between these two molecules, and found that neuronal knockdown of $f z$ in the fmi mutant $\operatorname{stan}^{f 00907}$ background showed comparable PD-Angle either to the $\operatorname{stan}^{f 00907}$ mutant itself or to neuronal knockdown of $f z$ in wild type background (Fig. $4 \mathrm{~g}$ ). We then performed the rescue experiments in $f z$ and fmi mutant background, respectively. Neuronal expression of $\mathrm{Fz}$ in the $\operatorname{stan}^{f 00907}$ mutant was able to rescue the dendrite phenotype to the degree of wild type (Fig. 4h). Similarly, in $f z^{E Y O 3114}$ mutant background, overexpression of Fmi in $\mathrm{ddaE}$ neuron also rescued the PD-Angle to the wild type degree (Fig. 4i). Thus, these results suggested that $\mathrm{Fz}$ and Fmi coordinate with each other in regulating directional growth of dendrites.

To determine whether $\mathrm{Fz}$ and Fmi function together in response to $\mathrm{Wg}$ signal, we performed genetic interaction experiments between either $\mathrm{Wg}$ and Fmi or Wg and Fz. Following knockdown of $f z$ in either $w g^{\text {spd-fg }}$ or $w g^{l-12}$ mutant background, we observed the PD-Angle did not further decrease in both situations. Notably, knockdown of $f z$ in a $w g^{s p d-f g}$ background resulted in a significant increase of the PD-Angle when compared to either the mutant or knockdown of $f z$ only, while the PD-Angle remained unchanged when knocked down $f z$ in $w g^{l-12}$ mutant background (Fig. 5a-d). In addition, the PD-Angle showed no further reduction following knockdown of $f m i$ in either $w g^{s p d-f g}$ or
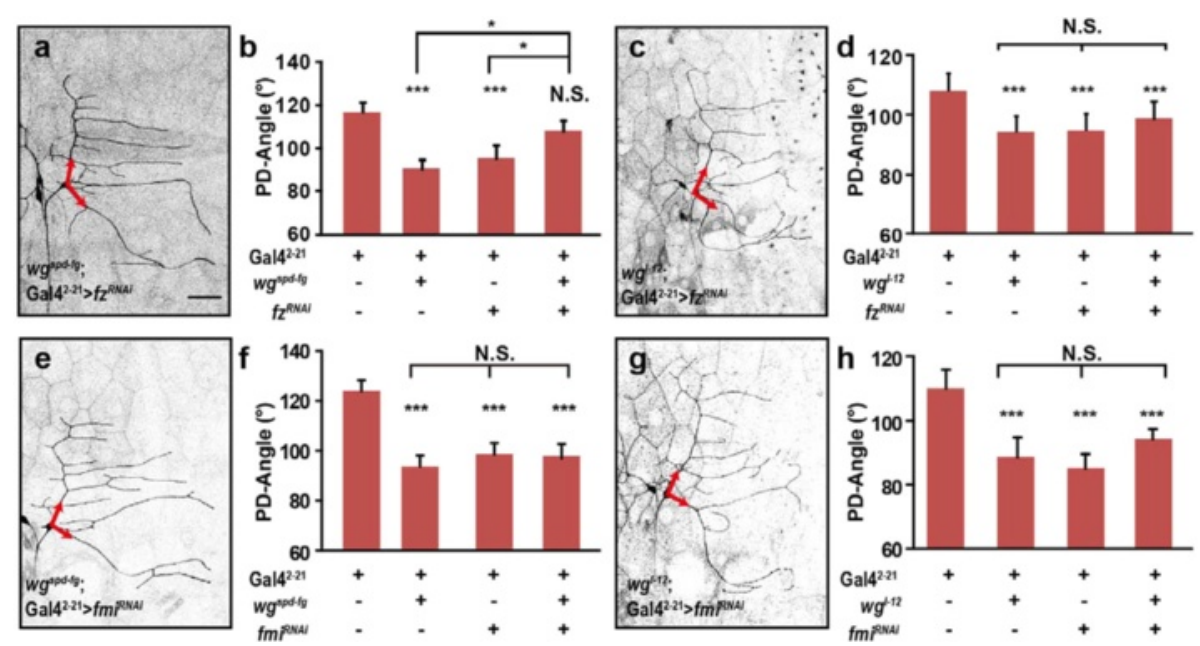

Fig. 5 Frizzled and flamingo mediates Wg signal to regulate dendrite directional growth in ddaE neuron. a-d Knockdown of $f z$ in $w g^{\text {spd-fg }}$ mutant background rescues the PD-Angle to wild type level, while knockdown of $f z$ in $w^{1-12}$ mutant background does not further decrease the PD-Angle. $(n=25-45)$. e-h Knockdown of fmi in a $w^{\text {spd-fg }}$ or wg $^{l-12}$ mutant background does not further decrease the PD-Angle. $(n=26-44)$. Red arrows indicate the initial parts of primary dendrites. Scale bar: $50 \mu \mathrm{m}$ 
$w g^{l-12}$ background (Fig. $5 \mathrm{e}-\mathrm{h}$ ). Together, these results suggested that neuronal $\mathrm{Fz}$ and $\mathrm{Fmi}$ cooperated in responding to environmental $\mathrm{Wg}$ and mediating the downstream events of Wg signal.

\section{Dishevelled and Rac1, but not main members of the $\beta$ -} catenin pathway, are required for conveying $\mathrm{Wg}$ signaling Dsh is a key molecule for both canonical and noncanonical Wnt signaling, where it functions downstream of $\mathrm{Fz}$ and Fmi $[42,43]$. Knockdown of $d s h$ in ddaE neuron led to a significant reduction in the PD-Angle, which was rescued by neuronal expressing Dsh (Fig. 6a-b). Thus, downstream of Fz and Fmi, Dsh is also required for the regulation of dendrite directional growth in ddaE neuron.

The canonical Wnt signal pathway is mediated by GSK3 $\beta$ and $\beta$-catenin [44], the Drosophila homologous of which are shaggy ( $\mathrm{sgg}$ ) and armadillo (arm), respectively. G protein Go mediates both Wg and PCP pathways transduced by $\mathrm{Fz}$ [45-47]. In addition, another $\mathrm{G}$ protein $\mathrm{Gq}$ has been suggested to couple with $\mathrm{Fz}$ in Drosophila [46] and also has been shown to function as a downstream molecule of Fmi intercellular signal in repressing dendrite growth [40]. However, we found that neither knockdown nor expressing a dominate-negative form of these proteins in ddaE neurons affected the PD-Angle, except for the forced expression of the transcription factor Arm, which could interfere with the expression of amount of Arm targeting genes (Additional file 7: Figure S7a-b). These results suggest that the canonical Wnt pathway is not required for mediating $\mathrm{Wg}$ signal in dendrite routing.

Small Rho GTPases have been shown to play critical roles in cytoskeleton assembly and neuronal morphogenesis $[48,49]$. We therefore examined whether Rac1, Cdc42, and Rho1 play a role in dendrite directional growth employing both gain-of and loss-of-function. The results showed that the PD-Angle was reduced when Rac1 was suppressed by neuronal expressing either $\operatorname{racl}^{R N A i}$ or $\mathrm{Rac1}^{\mathrm{T} 17 \mathrm{~N}}$ (a dominant negative form) (Fig. 6c-d). In contract, neither activating nor suppressing the activity of Cdc42 or/and Rho1 affect the PD-Angle (Additional file 7: Fig. S7c). Together, these results suggested that the small Rho GTPase Rac1 participates in the regulation of dendrite directional growth, which might function as a downstream factor in transducing the Wg signal to the cytoskeleton.

To examine whether Rac1 functions downstream of Wg signaling, we knocked down $r a c 1$ in either a $w g^{s p d-f g}$ or $w g^{l-12}$ mutant background. Knockdown of rac1 in $w g^{s p d-f g}$ background, led to a statistically increase of the PD-Angle when compared to either the mutant or knockdown of racl only, while the PD-Angle remained unchanged following knockdown of $\mathrm{racl}$ in a $\mathrm{wg}^{\mathrm{l}-12}$ background (Fig. 6e-h). These results suggested that in the $w g^{s p d-f g}$ mutant, at the position of ddaE neuron, the extracellular $\mathrm{Wg}$ distribution is changed, and down regulating rac1 may weaken the repulsive effect of $\mathrm{Wg}$ signal, resulting in a recovery of PD-Angle. Similar result was abstained from the interaction experiment of $w g^{s p d-f g}$ and $f z$. Furthermore, the PD-Angle remained unchanged following knockdown of racl in either fmi mutant $\operatorname{stan}^{f 00907}$ or $f z$ knockdown of background (Fig. 6i-j). Thus, these results suggest that Rac1 functions downstream of Fz and Fmi, thereby linking Wg signaling to the cytoskeleton, and this regulation is essential for proper directional growth of dendrites.

\section{Discussion}

The shape of dendrites is a major factor in determining both neuron morphology and the receptive field of dendrites, and therefore plays an important role in proper physiological functioning of neurons. Here, we report that epithelial Wg signal functions as a repulsive cue to control the growth direction of primary dendrites. We tested several Wg receptors and downstream molecules, and found that Fz, Fmi, Dsh, and Rac1 are essential for dendritic directional growth in response to Wg signals. The requirement of Rac1 suggests that the local regulation of cytoskeleton is required for dendrite routing. In contrast, the redundancy of $\beta$-catenin Wnt pathway suggests that the transcriptional control is not responsible for directional growth of dendrites (Fig. 6k). Our findings provide in vivo evidence that dendrites utilize Wnt signal as a spatial cue to generate a desired dendrite pattern.

\section{Wingless plays a repulsive role in the directional growth of primary dendrites}

The function of Wnt signaling in neuronal morphogenesis has been widely studied in the nervous system. Evolutionary conserved roles of Wnts are reported as both attractive and repulsive roles for regulating the direction of axon growth and guidance [21, 36, 50]. Wnt signal has been reported to promote dendrite branching and growth in cultured hippocampal neurons and C. elegans, respectively $[24,25]$. Nevertheless, the regulatory mechanisms of Wnt signaling in directional growth of dendrites have not been elucidated.

Here, we demonstrate that epithelial Wg provides repulsive cues for the primary dendrites of ddaE neuron. We employed two homozygous $w g$ mutants, $w g^{l-12}$ and $w g^{s p d-f g}$. Reduction in PD-Angle was observed in both of these two mutants. However, the Wg expression patterns in these two mutants are different. Wg expression was decreased in the posterior part in $w g^{l-12}$ mutant, and increased in the anterior part in $w g^{s p d-f g}$. In both situations, the change of the primary dendrites in direction relative to the body axes indicates that $\mathrm{Wg}$ signal functions as a repellent in regulating directional growth of dendrites, 


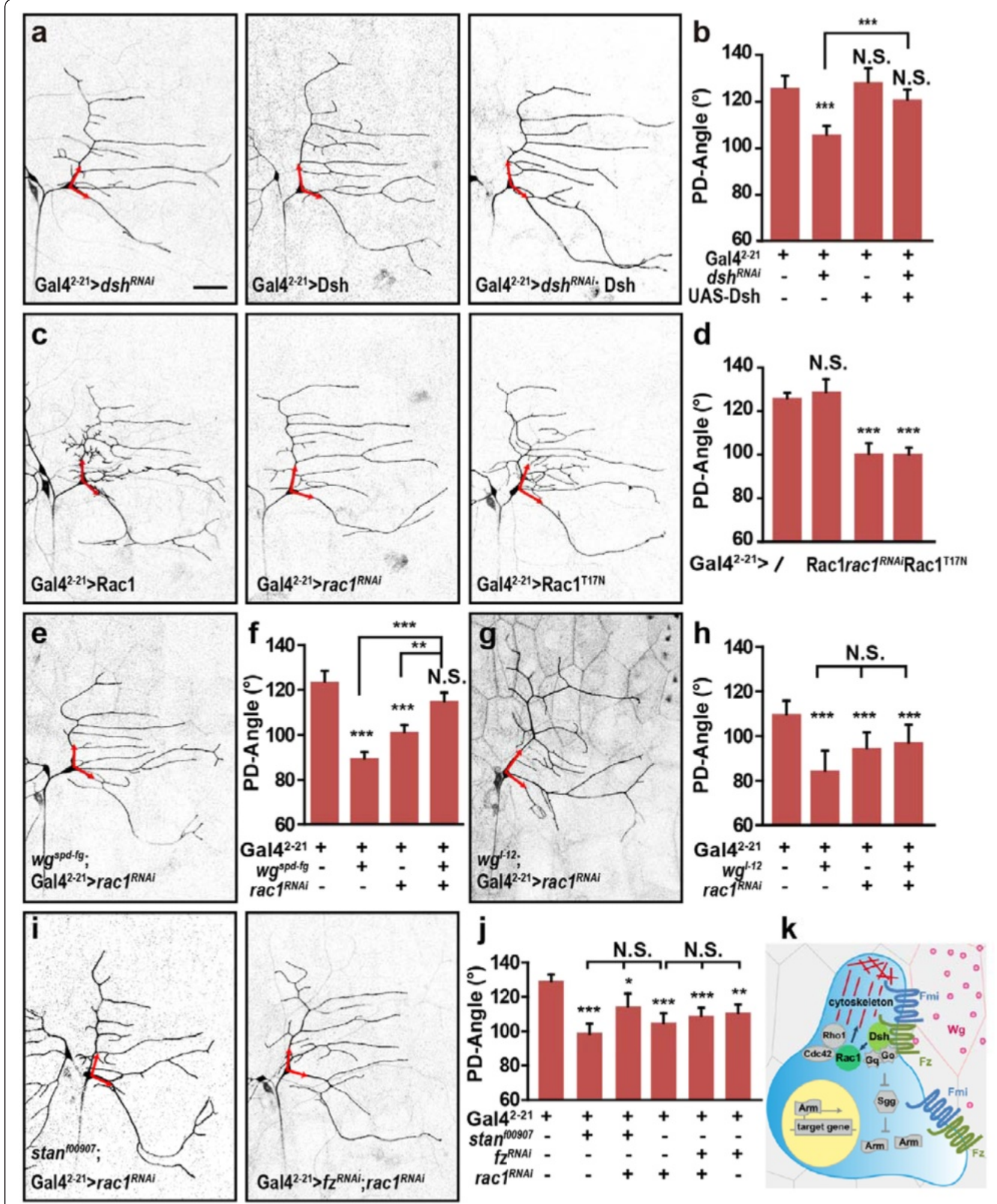

Fig. 6 (See legend on next page.) 
(See figure on previous page.)

Fig. 6 Dishevelled and Racl are involved in regulating dendrite orientation. $\mathbf{a}-\mathbf{b}$ Knockdown of dsh results in a significant reduction in the PD-Angle, which is rescued by expressing Dsh in the neuron. $(n=24-46)$. $\mathbf{c}-\mathbf{d}$ The PD-Angle is decreased when Rac1 is suppressed by expressing either $\mathrm{racl}^{\text {RNAi }}$ or Racl ${ }^{\text {T17N }}$ (a dominant negative form), whereas overexpressing Rac1 shows no effect on the PD-Angle. ( $n=38-53$ ). e-f Knockdown of racl partially rescues the reduced PD-Angle in $w^{\text {spd-fg }}$ mutant background. $(n=59-77)$. $\mathbf{g}-\mathbf{h}$ Knockdown racl does not further decrease the PD-Angle in wg $^{1-12}$ mutant background. $(n=24-36)$. i-j Knockdown of racl does not lead to further change of the PD-Angle in either fmi mutant stan ${ }^{\text {f0o907 }}$ or $\mathrm{fz}$ Knockdown of background. ( $n=37-76)$. $\mathbf{k}$ Schematic diagram of molecules in Wg signal pathway that regulates dendrite orientation. Fz, Fmi, Dsh, and Rac1 are essential for mediating Wg signal to regulate directional growth of dendrites, while G proteins, $\beta$-catenin pathway, and Cdc42/Rho1 are dispensable for the dendrite directional growth of ddaE neuron. Red arrows indicate the initial parts of primary dendrites. Scale bar: $50 \mu \mathrm{m}$

but not an inhibitor of general growth. Together, these results suggest that in addition to the function of Wnt signal in regulating axon guidance and dendrite outgrowth, this signal also serves as an important spatial cue for directional growth of dendrites.

\section{The directional growth of primary but not secondary dendrites of ddaE neuron is regulated by epithelial Wg signal}

The ddaE neuron features dendrites of comb-like pattern, with both primary and high-order dendrites extending in fixed directions. The cell-autonomous functions of various transcriptional factors in regulating the pattern of ddaE neuron have been studied in great details [51-53]. Environmental cues also participate in the dendrite patterning process. The neuron-glia interaction mediated by Neuroglian is critical for the formation of secondary order dendrites of ddaE neuron [54]. A recent study showed that during dendrite directional growth, Ten- $m$ is expressed in both neurons and epithelial cells, and the homodimer interaction guides the posterior extension of secondary dendrites according to the Ten-m gradient along the AP direction [17]. Our results showed that epithelia-derived Wg plays a repulsive role in regulating the routing of primary dendrites. Notably, although the primary dendrites grow outside Wg-expressing area, secondary dendrites grow straight through that region.

To understand the different responses between primary and secondary dendrites, we analyzed the detailed time window of $\mathrm{Wg}$ expression. The primary dendrites of ddaE neuron start sprouting at $13 \mathrm{~h}$ after egg laying (AEL), when Wg is still expressed in adjacent epithelial cells. In contrast, the secondary dendrites of ddaE neuron emerge at the end of embryogenesis, later than $15 \mathrm{~h}$ AEL, following the decay of Wg expression to levels below the detection limit. Thus, the time window of $\mathrm{Wg}$ expression allows its specific regulation in the directional growth of primary dendrites but not the secondary dendrites. Taken together with the findings that Ten-m signal specifically regulates the directional growth of high order dendrites, our findings show that epithelial signals line the dendrites at both DV and AP directions, and this regulation is precisely programmed during development.
Frizzled and Flamingo cooperate in mediating the $\mathbf{W g}$ signal in the regulation of dendrite directional growth The 7-transmembrane protein Fz and Fmi are core components of planar cell polarity (PCP) pathway. Previous studies indicate that Fz physically interacts with Fmi to mediate intercellular polarity signaling [37, 38]. Besides the function in PCP pathway, Fmi and Fz have also been found to play important roles in neural development. In studies on invertebrate, Fmi was reported to regulate axon and dendrite development in a Fz-independent manner [39, 40, 55-58]. In mammalian system, the interaction of $\mathrm{Fz}$ and Fmi was proposed to modulate the axon guidance in the commissural neurons and intrinsic enteric neurons $[59,60]$. Thus, whether these two molecules also function together in the nervous system remains unclear.

In this study, we demonstrate both $\mathrm{Fz}$ and Fmi functions in Wg signaling to regulate dendrite directional growth. Our results showed that simultaneous down regulation of both $f z$ and $f m i$ did not further decrease PD-Angle. Moreover, overexpression of Fz in $\operatorname{stan}^{f 00907}$ mutant background fully rescued the decreased PDAngle to the degree of wild type. In the reverse case, neural overexpression of Fmi in $f z^{E Y O 3114}$ mutant also rescued the mutant phenotype with no statistic difference to the wild type; however, the average PD-Angle in the rescue group was slightly smaller than wild type. Thus, we suspect that Fz, as a known Wg receptor, plays the key role in directing dendrite growth, while Fmi cooperates with $\mathrm{Fz}$ in this process. The rescue effect of Fmi overexpression may be based on the residue expression of $\mathrm{Fz}$ in $\mathrm{fz}^{\mathrm{EYO3114}}$ mutant (approximately $20 \%$ of wild type as shown in Fig. 4b). Previous studies have showed that in da neurons, Fmi regulates dendrite growth independent of $\mathrm{Fz}[39,40]$. Thus, our findings suggest that Fz-dependent function of Fmi specifically contributes to the control of directional growth but not general growth of dendrites.

\section{Directional growth of primary dendrites is regulated by non-canonical Wnt pathway through Dsh and Rac1}

As a key component of Wnt signaling, Dsh is required for mediating both the canonical $\beta$-catenin pathway and the non-canonical pathway. In this study, Dsh was also 
found to participate in the $\mathrm{Wg}$ signal-mediated directional growth of primary dendrites. Furthermore, we found that downstream of Fz-Fmi-Dsh, the canonical $\mathrm{Wnt} / \beta$-catenin pathway is dispensable for dendrite directional growth; instead, small GTPases Rac1 plays a critical role. It has been reported that Rac1 functions as a downstream factor in non-canonical Wnt pathway and regulates the dynamic of actin cytoskeleton in axon guidance and dendrite branching $[25,61,62]$. In particular, in response to Wnt signal, Rac1 has been found to directly bind to Dsh and regulate dendrite branching in cultured hippocampal neurons [25]. Remarkably, another two small GTPase, Cdc42 and Rho1, are not activated by Wnt7a in this response. In agreement, our in vivo results show that Rac1 is the effecter downstream of Wg signaling in dendrite routing, while Cdc42 and Rho1 are dispensable. Therefore, our results indicate that in the ddaE neuron, downstream of Fz-Fmi-Dsh, Rac1 conducts the Wg signaling to cytoskeleton, thereby regulating the directional growth of dendrites.

\section{Conclusions}

In conclusion, we established PD-Angle as a new parameter to analyze dendrite directional growth, and found that epithelial Wg serves as a repulsive cue that functions through Fz, Fmi, Dsh, and Rac1 to regulate the directional growth of primary dendrites. Thus, these findings gain insights into the non-cell-autonomous regulation by environmental cues of dendrite routing in neural development.

\section{Methods}

\section{Fly stocks}

All flies were maintained at $25^{\circ} \mathrm{C}$, except that flies containing $w g^{l-12}$ mutant were raised at $17^{\circ} \mathrm{C}$. Fly strains of Gal4 ${ }^{2-21}$, wg-Gal4, and elav-Gal4 were kindly provided by Dr. Fen-Biao Gao (University of Massachusetts Medical School). UAS-Fmi was a gift from Dr. Tadashi Uemura (Kyoto University). UAS-Go ${ }^{\text {GTP }}$ was from Dr. Andrew Tomlinson (Columbia University). UAS-Arm was generously provided by Dr. Haiyun Song (Shanghai Institutes for Biological Sciences, CAS). UAS-wntless-RNAi and UASvps26-RNAi were generously provided by Dr. Peng Zhang and Dr. Zengqiang Yuan (Institutes of Biophysics, CAS). The following fly strains were from the Bloomington stock center: fmi mutants $\operatorname{stan}^{f 00907}$ and $\operatorname{stan}^{f r z 3}$, wg mutants $w g^{s p d-f g}$ and $w g^{l-12}, f z$ mutant $f z^{E Y 03114}$, UAS-mCD8-eGFP, UAS-Dsh ${ }^{\text {myc }}$, UAS-Sgg ${ }^{\text {S9E }}$, UAS-Sgg ${ }^{\mathrm{B}}, \quad$ UAS-Rac1 ${ }^{\text {T17N }}$, UAS-Rac1, UAS-Rho1, UAS-Rho1 ${ }^{\text {GivV }}$, UAS-Rho1 ${ }^{\text {T19N }}$, UAS-Cdc $42^{\text {T17N }}$, UAS-Cdc 42 , UAS-Go $49 \mathrm{~B}^{\mathrm{R}}$, UASGa49B ${ }^{\text {dsRNA.1f1 }}$, UAS-Gq ${ }^{R N A i}$, UAS-fmi $i^{R N A i}$, UAS-rac1 ${ }^{R N A i}$, and UAS-cdc42 ${ }^{R N A i}$. The following RNAi fly lines were from Vienna Drosophila RNAi Center (VDRC): UAS- $w g^{R N A i}$, UAS- $d s h^{R N A i}$, UAS-sgg $g^{R N A i}$, and UAS-Go ${ }^{R N A i}$. UAS- $\operatorname{arm}^{R N A i-1}$ and UAS- $f z^{R N A i}$ were gifted from Dr. Jian-Quan Ni (Tsinghua Fly Center, School of Medicine, Tsinghua University). UAS-arm ${ }^{R N A i-2}$ was obtained from Fly Stocks of National Institute of Genetics (NIG-FLY).

The $w g^{s p d-f g_{-}}$Gal 4 construct was generated by subcloning a $9 \mathrm{~kb}$ DNA fragment upstream of the $w g$ coding region from the genomic DNA of the $w g^{\text {spd-fg }}$ mutant into a pPTGal vector [63]. The primers for the promoter amplification were 5'-AAAAGGCCTGTACTTTGAATCTTTCA CCTGCG-3', 5'-CGGGGTACCTATTGCTGATCGGGTT TATCTGTT-3'. We also used these primers to amplify the promoter of the $w^{1118}$ flies and generated the $w g^{w t}-$ Gal4 as a control.

\section{Immunohistochemistry}

Embryos and body walls of third instar larvae were immunostained according to the standard protocol [64] and was described in details in a previous research of our lab [40]. Samples were incubated in primary and then secondary antibodies at $4{ }^{\circ} \mathrm{C}$ over night. Rabbit-antiFz antibody (1:300) was kindly provided by Dr. David Strutt (University of Sheffield). Mouse monoclonal antibodies were purchased from Developmental Studies Hybridoma Bank (DSHB, University of Iowa): Wg (4D4, 1:250), Fmi (74-C, 1:100), 22C10 (1:500). Secondary antibodies were Alexa Fluor 555 Goat anti-mouse IgG (Invitrogen A21422, 1:1000), Alexa Fluor 488 Goat antirabbit IgG (Invitrogen A11008, 1:200) and Alexa Fluor ${ }^{\circ}$ 555 Goat anti-rabbit IgG (Invitrogen A21428, 1:200).

\section{Fluorescent In Situ Hybridization (FISH)}

Embryos of stage 13-14 were collected and fixed according to standard procedures [65]. The FISH was performed by using RNA scope based signal amplification (Advanced Cell Diagnostics). The $w g$ probe was designed to target the 479-1474 nt of $w g$ mRNA and labeled with $\mathrm{C} 2$ color channel. After rehydration, embryos were postfixed for $25 \mathrm{~min}$ by $5 \%$ formaldehyde in PBT and the protease digested using Protease III for $5 \mathrm{~min}$, following a second post-fixation. The probe hybridization was performed at $40^{\circ} \mathrm{C} \mathrm{O} / \mathrm{N}$, and the RNA signal was amplified by Amp1-4 at $40^{\circ} \mathrm{C}$. After each hybridization step, embryos were washed by $0.02 \%$ SSCT. Amp 4 Alt A-FL was used for the fluorescent labeling.

\section{Imaging and analysis}

VECTASHIELD ${ }^{\circ}$ mounting medium (Vector Laboratories) was used to mount samples after immunostaining. Third instar larvae were rinsed in PBS and then immobilized using a cover slip for in vivo imaging. Confocal images were obtained with a Leica SPE or a SP5 II MP microscope or a Nikon A1 confocol. The dendrites morphology was analyzed using NIS-Element D 3.0 (Nikon) and a self- 
developed program, and the fluorescent intensity was measured by Image J (National Institutes of Health, USA).

The parameters for estimating the dendrite morphology of ddaE neuron were defined as: 1) PD-Angle, the angle between two primary dendrites, which is determined by initial $20 \mu \mathrm{m}$ primary dendrites; 2) the initial parts of primary dendrites, the trajectory of primary dendrites of ddaE neuron was traced by Image and plotted by $R$ project ( $R$ Development Core Team), the 20 $\mu \mathrm{m}$ points were dotted by ImageJ and plotted in Excel (Microsoft), and the coordinate of average direction of dorsal or ventral primary was the average of the coordinates of all dorsal or ventral intersections; 3) PD-Area, the dendrite area outlined by the primary dendrites; 4 ) Total area, the dendrite area outlined by all dendritic tips; 5) The dendritic coverage range at AP direction and at DV direction; 6) PD-Total length, the total length of the two primary dendrites. Neurons that were not able to establish the entire primary dendrites were not included in the data analysis.

To examine the expression of Wg signal, we conducted the following experiments: 1 ) Wg-expressing cells were visualized using UAS-mCD8-eGFP driven by $w g$-Gal4 or $w g^{s p d-f g_{-G a l}}$ or $w g^{w t}$-Gal4 signal, and neurons were stained by $22 \mathrm{C} 10$ antibody. In Fig. $1 \mathrm{~b}$ and Additional file 3: Fig. S3a, 25 images of AS4-6 and 20 images of TS2-3 were obtained from $10 w g$-Gal4 > GFP embryos. In Additional file 3: Fig. S3c, 18 images of AS4-6 were obtained from $10 w g^{\text {spd-fg }}$-Gal $4>$ GFP or $w g^{w t}{ }_{-G a l}$-G $>$ GFP embryos. All images were rotated to anterior-left and dorsal-up and were aligned by having ddaE neuron on the origin of the coordinate. The gray value of GFP signal was read, averaged and calculated to generate pseudo-color figures by Matlab (MathWorks, Natick, MA). Showing in Fig. $1 \mathrm{~b}$ and Additional file 3: Fig. S3, different colors represented the corresponding brightness as the bar indicated. 2) Intensity of Wg, after standard Wg antibody staining and confocal imaging procedure, the fluorescent intensity in a $10 \times 30 \mu^{2}$ region covering all $\mathrm{Wg}$ expressing cells in the dorsal side of a semi-segment is measured and averaged by Image J; 3) Center-to-boundary Distance, the distance between the center of Wg or $w g$ RNA signal and the posterior segmental boundary, which is normalized to segment width; 4) $w g$ distribution, the range of $w g$ mRNA distribution at A-P axis normalized to segment width. During the measurement of the parameter Center-to-boundary and $w g$ distribution, the genotypes were renumbered and blinded to the operator.

Data in this paper met to normal distribution. Statistical analysis was performed using two tails Student's t-test and one-way analysis of variance (ANOVA) with Tukey's test as a post hoc comparison. Quantification data are shown in mean \pm s.e.m; ${ }^{*} P<0.05,{ }^{* *} P<0.01$, ${ }^{* * *} P<0.001$, and N.S. represents no significant.

\section{Quantitative real time PCR}

Total RNA was extracted from approximate $50 \mathrm{ul} \mathrm{em-}$ bryos at embryonic stage 14 with TRIzol (Invitrogen). $1 \mu \mathrm{g}$ of RNA samples were treated with RQ1 DNase (Promega) and reverse transcribed using PrimeScript RT Master Mix (TaKaRa). Relative quantification PCR was carried out using a SYBR Premix Ex TaqTM II kit (Takara) and an ABI PRISM 7300 real-time PCR Detection system (Applied Biosystems). Relative mRNA levels were calculated using the comparative CT method. Rp49 was used as an internal control, and gene expression levels were normalized to treatment control or genetic control. Three separate samples were collected from each condition, and measurements were conducted in triplicates. The primers of $f z$ for q-PCR were $f z$-qPCR-exon1-F: TGCAACTGAAAACGCCTCT; $f z$-qPCR-exon2-R: AAA CGGCCAA GAAGACAATG. The primers of $r p 49$ were rp49-RT-F: AGGGTATCGACAACAGAGTG; rp49-RT-R: CACCAGGAACTTCTTGAATC

\section{Ethics approval and consent to participate}

The research does not involve in any human subjects. All the animal experiments are in accordance with ethical principles of Basel Declaration and ethical guidelines of the International Council for Laboratory Animal Science.

\section{Statement of consent for publication}

This manuscript contains no individual person's data. The statement of consent for publication is not applicable in this article.

\section{Availability of data and materials}

The datasets supporting the conclusions of this article are included within the article and its additional files.

\section{Additional file}

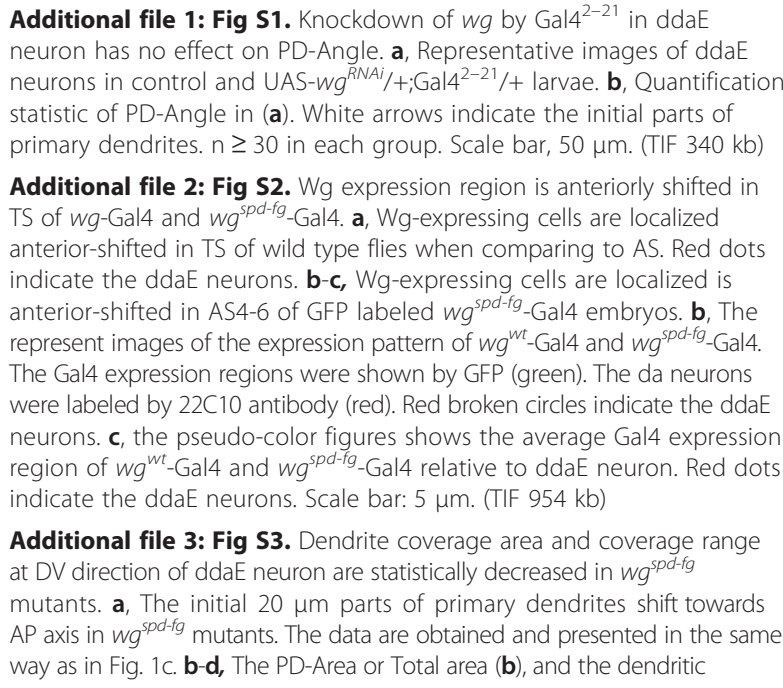

Additional file 3: Fig S3. Dendrite coverage area and coverage range at DV direction of ddaE neuron are statistically decreased in $w^{\text {spd-fg }}$

mutants. a, The initial $20 \mu \mathrm{m}$ parts of primary dendrites shift towards AP axis in $w g^{\text {spd-fg }}$ mutants. The data are obtained and presented in the same way as in Fig. 1c. b-d, The PD-Area or Total area (b), and the dendritic 
coverage range at DV direction (c) are significantly decreased in $\mathrm{wg}^{\mathrm{spd} f \mathrm{fg}}$ mutants, while the PD-Total length (d) and the dendritic coverage range at AP direction (c) are unchanged. The insert cartoons show dendrites in green lines and the parameter quantified in corresponding panels. ( $n=42-43$ ). (TIF $399 \mathrm{~kb})$

Additional file 4: Fig S4. Fz was expressed in $\mathrm{ddaE}$ and the expression level decreased in $\mathrm{fZ}^{\mathrm{FO} O 3114}$ mutant. a, Fz expression is detected in ddaE neuron in wild type S14 embryos. Fz protein is shown by anti-Fz antibody (green) and neurons are shown by antibody $22 \mathrm{C} 10$ (red). b, Fz is co-expressed with Fmi in dorsal cluster da neurons. Fz protein is shown by anti-Fz antibody (green) and neurons are shown by anti-Fmi antibody (red). Scale bar: $5 \mu \mathrm{m}$. (TIF $1.11 \mathrm{mb}$ )

Additional file 5: Fig S5. The PD-angle is not affected in RYK-family receptor drl, drl-2, or dnt mutant or knock-down flies. $\mathbf{a}-\mathbf{b}$, No significant change of PD-Angle is observed in drl, drl-2, or dnt mutants. c-d, Neuronal overexpression or knock-down of either $d r l$ or $d r l-2$ has no effect on the PD-Angle. White arrows indicate the initial parts of primary dendrites. $n \geq 30$ in each group. Scale bar, $50 \mu \mathrm{m}$. (TIF $1.44 \mathrm{mb}$ )

Additional file 6: Fig S6. Flamingo is required in ddaE neuron for dendrite directional growth. $\mathbf{a}$, In homozygous available $\operatorname{stan}^{f r z 3}$ mutant, Fmi expression is decreased in epithelia and da neurons. $\mathbf{b}-\mathbf{c}$, The PDAngle is significantly decreased in $\operatorname{stan}^{\text {frz }}$ mutant. White arrows indicate the initial parts of primary dendrites. $n \geq 30$ in each group. Scale bar, 5 $\mu \mathrm{m}$ in (a) and $50 \mu \mathrm{m}$ in (b). (TIF $1.05 \mathrm{mb}$ )

Additional file 7: Fig S7. Canonical-Wnt pathway and small GTPase Cdc42, Rho1 were unaffected in dendrite directional growth. a, Neither knockdown nor overexpression of Go or Gq in ddaE neurons has an effect on the PD-Angle. $\mathbf{b}$, Neither knockdown nor overexpression of Sgg or Arm in ddaE neurons has an effect on the PD-Angle, except that overexpressing Arm results in a significant decrease. $\mathbf{c}$, No significant change of the PD-Angle is shown when manipulating Cdc42 or/and Rho1 in ddaE neuron. CA, constitutively active form; $O E$, overexpression; DN, dominant negative form. $n \geq 30$ in each group. (TIF $349 \mathrm{~kb}$ )

\section{Abbreviations}

AEL: after egg laying: AP: anterior-posterior; Arm: Armadillo; AS: abdominal segments; BDNF: Brain-derived neurotrophic factor; Center-to-boundary Distance: the distance between the center of wg distribution and the posterior segmental boundary; da: dendritic arborization; Dnt: Doughnut; Drl: Derailed; Drl-2: Derailed 2; Dsh: Dishevelled; DV: dorsal-ventral; ECM: extracellular matrix; FISH: fluorescent in situ hybridization; Fmi: Flamingo; Fz: Frizzled; PCP: planar cell polarity; PD-Angle: the angle between the initial $20 \mu \mathrm{m}$ of two primary dendrites; PD-Area: the dendrite coverage area outlined by the two primary dendrites; PD-Total length: the total length of the two primary dendrites; Sema3A: Semaphorin3A: Sgg: Shaggy; Total area: the dendrite coverage area outlined by all branch tips; TS: thoracic segments; Wg: Wingless; wg distribution: the width of wg distribution region; Wls: Wntless.

\section{Competing interests}

The authors declare that they have no competing interests.

\section{Authors' contributions}

$X L, Y W$, and $Y L$ designed the experiments and drafted the manuscript. $Y W$, $X L, H W, T L, W Y$, and JG performed the experiments and analyzed the data. All authors read and approved the final manuscript.

\section{Acknowledgements}

We are grateful to Dr. Fen-Biao Gao, Dr. Jianquan Ni, Dr. Tadashi Uemura, Dr. Haiyun Song, Dr. Andrew Tomlinson, Dr. Peng Zhang, and Dr. Zengqiang Yuan for generously providing fly strains, and We are also grateful to Dr. David Strutt for providing the Fz antibody. We thank Junfeng Hao (Institute of Biophysics, Chinese Academy of Sciences) for the technical support in the experiment of RNA scope. We thank the Bloomington Stock Center, Vienna Drosophila RNAi Center (VDRC), Fly Stocks of National Institute of Genetics (NIG-FLY), and the Tsinghua Fly Center for kindly providing fly lines. We thank Jingwu Hou (Institute of Biophysics, Chinese Academy of Sciences) for his excellent technical support and Bangyu Zhou (Institute of Neuroscience, Chinese Academy of Sciences) for the software programming.

\section{Funding}

This work was supported by the National Science Foundation of China (31070956, 91132709, and 31571089) and the One Hundred Talents Project of the CAS (KSCX2-YW-R-156).

\section{Author details}

${ }^{1}$ State Key Laboratory of Brain and Cognitive Science, Institute of Biophysics, Chinese Academy of Sciences, Beijing 100101, China. 'University of Chinese Academy of Sciences, Beijing 100049, China. ${ }^{3}$ Beijing Institutes of Life Science, Chinese Academy of Sciences, Beijing 100101, China.

Received: 12 January 2016 Accepted: 21 April 2016

Published online: 29 April 2016

\section{References}

1. Dong X, Shen K, Bulow HE. Intrinsic and extrinsic mechanisms of dendritic morphogenesis. Annu Rev Physiol. 2015;77:271-300.

2. Corty MM, Matthews BJ, Grueber WB. Molecules and mechanisms of dendrite development in Drosophila. Development. 2009;136(7):1049-61.

3. Jan YN, Jan LY. Branching out: mechanisms of dendritic arborization. Nat Rev Neurosci. 2010;11(5):316-28.

4. Campbell DS, Stringham SA, Timm A, Xiao T, Law MY, Baier H, Nonet ML, Chien CB. Slit1a inhibits retinal ganglion cell arborization and synaptogenesis via Robo2-dependent and -independent pathways. Neuron. 2007;55(2):231-45.

5. Dijkhuizen PA, Ghosh A. BDNF regulates primary dendrite formation in cortical neurons via the PI3-kinase and MAP kinase signaling pathways. J Neurobiol. 2005;62(2):278-88.

6. Polleux F, Morrow T, Ghosh A. Semaphorin $3 \mathrm{~A}$ is a chemoattractant for cortical apical dendrites. Nature. 2000;404(6778):567-73.

7. Šestan N, Artavanis-Tsakonas S, Rakic P. Contact-dependent inhibition of cortical neurite growth mediated by notch signaling. Science. 1999; 286(5440):741-6.

8. Dong X, Liu OW, Howell AS, Shen K. An extracellular adhesion molecule complex patterns dendritic branching and morphogenesis. Cell. 2013;155(2):296-307.

9. Salzberg Y, Diaz-Balzac CA, Ramirez-Suarez NJ, Attreed M, Tecle E, Desbois M, Kaprielian Z, Bulow HE. Skin-derived cues control arborization of sensory dendrites in Caenorhabditis elegans. Cell. 2013;155(2):308-20.

10. Komiyama T, Sweeney LB, Schuldiner O, Garcia KC, Luo L. Graded expression of semaphorin-1a cell-autonomously directs dendritic targeting of olfactory projection neurons. Cell. 2007;128(2):399-410.

11. Furrer MP, Kim S, Wolf B, Chiba A. Robo and Frazzled/DCC mediate dendritic guidance at the CNS midline. Nat Neurosci. 2003;6(3):223-30.

12. Han $C$, Wang $D$, Soba $P$, Zhu $S$, Lin $X$, Jan LY, Jan $Y N$. Integrins regulate repulsion-mediated dendritic patterning of drosophila sensory neurons by restricting dendrites in a 2D space. Neuron. 2012;73(1):64-78.

13. Kim ME, Shrestha BR, Blazeski R, Mason CA, Grueber WB. Integrins establish dendrite-substrate relationships that promote dendritic self-avoidance and patterning in drosophila sensory neurons. Neuron. 2012;73(1):79-91.

14. Jiang N, Soba P, Parker E, Kim CC, Parrish JZ. The microRNA bantam regulates a developmental transition in epithelial cells that restricts sensory dendrite growth. Development. 2014;141(13):2657-68.

15. Parrish JZ, Xu P, Kim CC, Jan LY, Jan YN. The microRNA bantam functions in epithelial cells to regulate scaling growth of dendrite arbors in drosophila sensory neurons. Neuron. 2009;63(6):788-802.

16. Song Y, Ori-McKenney KM, Zheng Y, Han C, Jan LY, Jan YN. Regeneration of Drosophila sensory neuron axons and dendrites is regulated by the Akt pathway involving Pten and microRNA bantam. Genes Dev. 2012;26(14):1612-25.

17. Hattori Y, Usui T, Satoh D, Moriyama S, Shimono K, Itoh T, Shirahige K, Uemura T. Sensory-neuron subtype-specific transcriptional programs controlling dendrite morphogenesis: genome-wide analysis of Abrupt and Knot/Collier. Dev Cell. 2013;27(5):530-44.

18. Ciani L, Salinas PC. WNTs in the vertebrate nervous system: from patterning to neuronal connectivity. Nat Rev Neurosci. 2005;6(5):351-62.

19. Park M, Shen K. WNTs in synapse formation and neuronal circuitry. EMBO J. 2012;31(12):2697-704.

20. Rosso SB, Inestrosa NC. WNT signaling in neuronal maturation and synaptogenesis. Front Cell Neurosci. 2013;7:103.

21. Bovolenta P, Rodriguez J, Esteve P. Frizzled/RYK mediated signalling in axon guidance. Development. 2006;133(22):4399-408.

22. van Amerongen $R$, Nusse $R$. Towards an integrated view of Wnt signaling in development. Development. 2009;136(19):3205-14. 
23. Wu Y, Helt JC, Wexler E, Petrova IM, Noordermeer JN, Fradkin LG, Hing H. Wnt5 and Drl/Ryk Gradients Pattern the Drosophila Olfactory Dendritic Map. J Neurosci. 2014;34(45):14961-72.

24. Kirszenblat L, Pattabiraman D, Hilliard MA. LIN-44/Wnt directs dendrite outgrowth through LIN-17/Frizzled in C. elegans Neurons. PLoS Biol. 2011;9(9):e1001157.

25. Rosso SB, Sussman D, Wynshaw-Boris A, Salinas PC. Wnt signaling through Dishevelled, Rac and JNK regulates dendritic development. Nat Neurosci. 2005:8(1):34-42

26. Couso JP, Bishop SA, Martinez Arias A. The wingless signalling pathway and the patterning of the wing margin in Drosophila. Development. 1994;120(3):621-36.

27. van den Heuvel M, Harryman-Samos C, Klingensmith J, Perrimon N, Nusse R. Mutations in the segment polarity genes wingless and porcupine impair secretion of the wingless protein. EMBO J. 1993;12(13):5293-302.

28. Sugimura K, Yamamoto M, Niwa R, Satoh D, Goto S, Taniguchi M, Hayashi S, Uemura T. Distinct developmental modes and lesion-induced reactions of dendrites of two classes of Drosophila sensory neurons. J Neurosci. 2003;23(9):3752-60

29. Bänziger C, Soldini D, Schütt C, Zipperlen P, Hausmann G, Basler K. Wntless, a conserved membrane protein dedicated to the secretion of Wnt proteins from signaling cells. Cell. 2006;125(3):509-22.

30. Port F, Kuster M, Herr P, Furger E, Banziger C, Hausmann G, Basler K. Wingless secretion promotes and requires retromer-dependent cycling of Wntless. Nat Cell Biol. 2008;10(2):178-85.

31. Neumann CJ, Cohen SM. Distinct mitogenic and cell fate specification functions of wingless in different regions of the wing. Development. 1996;122(6):1781-9.

32. Seifert JR, Mlodzik M. Frizzled/PCP signalling: a conserved mechanism regulating cell polarity and directed motility. Nat Rev Genet. 2007;8(2):126-38.

33. Bellen HJ, Levis RW, Liao G, He Y, Carlson JW, Tsang G, Evans-Holm M, Hiesinger PR, Schulze KL, Rubin GM, et al. The BDGP gene disruption project: single transposon insertions associated with $40 \%$ of Drosophila genes. Genetics. 2004;167(2):761-81.

34. Fradkin LG, Dura JM, Noordermeer JN. Ryks: new partners for Wnts in the developing and regenerating nervous system. Trends Neurosci. 2010;33(2):84-92.

35. Liu Y, Shi J, Lu CC, Wang ZB, Lyuksyutova Al, Song XJ, Zou Y. Ryk-mediated Wnt repulsion regulates posterior-directed growth of corticospinal tract. Nat Neurosci. 2005;8(9):1151-9.

36. Yoshikawa S, McKinnon RD, Kokel M, Thomas JB. Wnt-mediated axon quidance via the Drosophila Derailed receptor. Nature. 2003;422(6932):583-8.

37. Chen WS, Antic D, Matis M, Logan CY, Povelones M, Anderson GA, Nusse R, Axelrod JD. Asymmetric homotypic interactions of the atypical cadherin flamingo mediate intercellular polarity signaling. Cell. 2008;133(6):1093-105.

38. Usui T, Shima Y, Shimada Y, Hirano S, Burgess RW, Schwarz TL, Takeichi M, Uemura T. Flamingo, a seven-pass transmembrane cadherin, regulates planar cell polarity under the control of Frizzled. Cell. 1999;98(5):585-95.

39. Gao FB, Kohwi M, Brenman JE, Jan LY, Jan YN. Control of dendritic field formation in Drosophila: the roles of flamingo and competition between homologous neurons. Neuron. 2000;28(1):91-101.

40. Wang Y, Wang H, Li X, Li Y. Epithelial microRNA-9a regulates dendrite growth through Fmi-Gq signaling in Drosophila sensory neurons. Dev Neurobiol. 2016; 76(2):225-37.

41. Rawls AS, Wolff T. Strabismus requires Flamingo and Prickle function to regulate tissue polarity in the Drosophila eye. Development. 2003:130(9):1877-87.

42. Shimada Y, Usui T, Yanagawa S, Takeichi M, Uemura T. Asymmetric colocalization of Flamingo, a seven-pass transmembrane cadherin, and Dishevelled in planar cell polarization. Curr Biol. 2001;11(11):859-63.

43. Song S, Zhang B, Sun H, Li X, Xiang Y, Liu Z, Huang X, Ding M. A Wnt-Frz/ Ror-Dsh pathway regulates neurite outgrowth in Caenorhabditis elegans. PLoS Genet. 2010:6(8):e1001056.

44. Clevers H, Nusse R. Wnt/beta-catenin signaling and disease. Cell. 2012; 149(6):1192-205

45. Katanaev VL, Ponzielli R, Semeriva M, Tomlinson A. Trimeric G proteindependent frizzled signaling in Drosophila. Cell. 2005;120(1):111-22.

46. Koval A, Purvanov V, Egger-Adam D, Katanaev VL. Yellow submarine of the Wnt/Frizzled signaling: submerging from the $\mathrm{G}$ protein harbor to the targets. Biochem Pharmacol. 2011;82(10):1311-9.

47. Luchtenborg AM, Solis GP, Egger-Adam D, Koval A, Lin C, Blanchard MG, Kellenberger S, Katanaev VL. Heterotrimeric Go protein links Wnt-Frizzled signaling with ankyrins to regulate the neuronal microtubule cytoskeleton. Development. 2014;141(17):3399-409.
48. Luo L. Actin cytoskeleton regulation in neuronal morphogenesis and structural plasticity. Annu Rev Cell Dev Biol. 2002;18:601-35.

49. Ng J, Nardine T, Harms M, Tzu J, Goldstein A, Sun Y, Dietzl G, Dickson BJ, Luo L. Rac GTPases control axon growth, guidance and branching. Nature. 2002;416(6879):442-7.

50. Paganoni S, Ferreira A. Neurite extension in central neurons: a novel role for the receptor tyrosine kinases Ror1 and Ror2. J Cell Sci. 2005;118(Pt 2):433-46.

51. Li W, Wang F, Menut L, Gao FB. BTB/POZ-zinc finger protein abrupt suppresses dendritic branching in a neuronal subtype-specific and dosage-dependent manner. Neuron. 2004;43(6):823-34.

52. Parrish JZ, Kim MD, Jan LY, Jan YN. Genome-wide analyses identify transcription factors required for proper morphogenesis of Drosophila sensory neuron dendrites. Genes Dev. 2006;20(7):820-35.

53. Sugimura K, Satoh D, Estes P, Crews S, Uemura T. Development of morphological diversity of dendrites in Drosophila by the BTB-zinc finger protein abrupt. Neuron. 2004;43(6):809-22.

54. Yamamoto M, Ueda R, Takahashi K, Saigo K, Uemura T. Control of axonal sprouting and dendrite branching by the Nrg-Ank complex at the neuron-glia interface. Curr Biol. 2006;16(16):1678-83.

55. Huarcaya Najarro E, Ackley BD. C. elegans fmi-1/flamingo and Wnt pathway components interact genetically to control the anteroposterior neurite growth of the VD GABAergic neurons. Dev Biol. 2013:377(1):224-35.

56. Kimura H, Usui T, Tsubouchi A, Uemura T. Potential dual molecular interaction of the Drosophila 7-pass transmembrane cadherin Flamingo in dendritic morphogenesis. J Cell Sci. 2006;119(Pt 6):1118-29.

57. Matsubara D, Horiuchi SY, Shimono K, Usui T, Uemura T. The seven-pass transmembrane cadherin Flamingo controls dendritic self-avoidance via its binding to a LIM domain protein, Espinas, in Drosophila sensory neurons. Genes Dev. 2011;25(18):1982-96.

58. $\mathrm{Ng} J$. Wnt/PCP proteins regulate stereotyped axon branch extension in Drosophila. Development. 2012;139(1):165-77.

59. Sasselli V, Boesmans W, Vanden Berghe P, Tissir F, Goffinet AM, Pachnis V. Planar cell polarity genes control the connectivity of enteric neurons. J Clin Invest. 2013;123(4):1763-72.

60. Shafer B, Onishi K, Lo C, Colakoglu G, Zou Y. Vangl2 promotes Wnt/planar cell polarity-like signaling by antagonizing Dvl1-mediated feedback inhibition in growth cone guidance. Dev Cell. 2011;20(2):177-91.

61. Srahna M, Leyssen M, Choi CM, Fradkin LG, Noordermeer JN, Hassan BA. A signaling network for patterning of neuronal connectivity in the Drosophila brain. PLoS Biol. 2006;4(11):e348.

62. Tsuji T, Ohta Y, Kanno Y, Hirose K, Ohashi K, Mizuno K. Involvement of p114-RhoGEF and Lfc in Wnt-3a- and dishevelled-induced RhoA activation and neurite retraction in N1E-115 mouse neuroblastoma cells. Mol Biol Cell. 2010:21(20):3590-600.

63. Sharma Y, Cheung U, Larsen EW, Eberl DF. PPTGAL, a convenient Gal4 P-element vector for testing expression of enhancer fragments in drosophila. Genesis (New York, NY: 2000). 2002;34(1-2):115.

64. Wu JS, Luo L. A protocol for dissecting Drosophila melanogaster brains for live imaging or immunostaining. Nat Protoc. 2006;1(4):2110-5.

65. Kosman D, Mizutani CM, Lemons D, Cox WG, McGinnis W, Bier E. Multiplex detection of RNA expression in Drosophila embryos. Science. 2004;305(5685):846

\section{Submit your next manuscript to BioMed Central and we will help you at every step:}

- We accept pre-submission inquiries

- Our selector tool helps you to find the most relevant journal

- We provide round the clock customer support

- Convenient online submission

- Thorough peer review

- Inclusion in PubMed and all major indexing services

- Maximum visibility for your research

Submit your manuscript at www.biomedcentral.com/submit 